\title{
Propriedades físicas e mecânicas da madeira de Pinus palustris Mill. cultivado no sul do Brasil
}

\author{
Annah Carolina Bajaluk Bilik ${ }^{1 *}$, Josiane Fernandes Keffer $^{1}$, Rosilani Trianoski ${ }^{1}$
}

\begin{abstract}
RESUMO: Plantios experimentais de Pinus palustris foram implantados no Brasil com a finalidade de identificar a região mais adequada para o desenvolvimento da espécie. No entanto, a aceitabilidade da madeira dessa espécie no mercado interno depende do conhecimento de suas propriedades básicas. Por isso, este trabalho teve como objetivo determinar algumas das propriedades físicas e mecânicas da madeira de P. palustris cultivado no sul do Brasil. Para tanto, foram extraídas amostras de dez árvores com 39 anos de idade para a realização dos ensaios. A densidade da madeira variou de 0,35 a $0,92 \mathrm{~g} \cdot \mathrm{cm}^{-3}$ nas condições básica e verde, respectivamente. As contrações tangencial e radial resultaram num coeficiente de retratibilidade de 1,85. Apesar da idade avançada, a resistência mecânica da madeira de $P$. palustris foi semelhante à de outras espécies do mesmo gênero. Conclui-se que a espécie apresenta madeira com propriedades que a tornam potencial para diversificação dos plantios comerciais da região sul brasileira.
\end{abstract}

Palavras-chave: pinheiro de folha longa, resistência da madeira, estabilidade dimensional, qualidade da madeira

\section{Physical and mechanical properties of Pinus palustris Mill. wood grown in southern Brazil}

\begin{abstract}
Experimental plantations of Pinus palustris were implanted in Brazil with the objective of identifying the most suitable region for the development of the species. However, the acceptability of wood of this species in the national market depends on the knowledge of its basic properties. Therefore, this work aimed to determine some of the physical and mechanical properties of the P. palustris wood grown in southern Brazil. For this purpose, samples were taken from ten 39-year-old trees for testing. The wood density varied from 0.35 to $0.92{\mathrm{~g} . \mathrm{cm}^{-3}}^{-}$in the basic and green conditions, respectively. The tangential and radial contractions resulted in a retractability coefficient of 1.85 . Despite the advanced age, the mechanical resistance of $P$. palustris wood was similar to that of other species of the same genus. It is concluded that the species has wood with properties that make it potential for diversification of commercial plantations in the southern region of Brazil.
\end{abstract}

Keywords: long leaf pine, wood strength, dimensional stability, wood quality

\section{INTRODUÇÃO}

O pinheiro de folha longa (Pinus palustris Mill.) é uma das principais espécies de pinus do sul dos Estados Unidos (SHIMIZU, 1979), e suas principais vantagens são a capacidade de crescer em solos arenosos e de baixa fertilidade, resistência ao fogo, tolerância à seca e baixa demanda de nutrientes (BERENHAUSER, 1971; DORMAN, 1976; BOYER, 1990). Devido às dificuldades de implantação, principalmente nos primeiros anos, quando o crescimento fica estagnado, essa espécie não foi plantada no Brasil na mesma escala que o $P$. elliottii e o P. taeda (SHIMIZU, 1979). Apesar disso, este autor defende que o $P$. palustris pode servir como uma espécie alternativa para a diversificação dos reflorestamentos da região sul brasileira, por isso, precisa ser melhor investigada.

Historicamente, a madeira dessa espécie era a preferida para construção de casas, vigas de pontes e construção naval, além de ser uma excelente produtora de resina, o que confere maior durabilidade à madeira (BERENHAUSER, 1971). Por isso, ele considera a espécie promissora para a coleta de resina e produção de madeira estrutural. No entanto, para que a madeira dessa espécie seja aceita no mercado interno, é necessário o conhecimento de suas propriedades físicas e mecânicas.

As propriedades físicas e mecânicas das madeiras são importantes para a identificação do seu potencial de utilização, visto que é com base nessas características que são definidas as aplicações em que serão empregadas (LATORRACA, ALBUQUERQUE, 2000; MORAES NETO et al., 2009). Portanto, o objetivo deste trabalho foi determinar as propriedades físicas (densidade e retratibilidade) e mecânicas (módulo de ruptura (MOR) e módulo de elasticidade (MOE) à flexão estática) da madeira de $P$. palustris cultivado na região sul brasileira.

\section{MATERIAL E MÉTODOS}

Para o desenvolvimento desta pesquisa foram amostradas 10 árvores de $P$. palustris com 39 anos de idade provenientes de um plantio experimental na Estação Experimental Rio Negro da Universidade

\footnotetext{
${ }^{1}$ Universidade Federal do Paraná

* Email: annahbilik@gmail.com
} 
Federal do Paraná, localizada no município de Rio Negro, cujas coordenadas geográficas centrais são: latitude $26^{\circ} 03^{\prime} 43^{\prime \prime} \mathrm{S}$ e longitude $49^{\circ} 45^{\prime} 32^{\prime \prime} \mathrm{W}$ e altitude de $800 \mathrm{~m}$. O clima da região segundo classificação de Köppen é o $\mathrm{Cfb}$, tipo climático temperado propriamente dito, com temperatura média do mês mais frio menor que $18{ }^{\circ} \mathrm{C}$ e temperatura média do mês mais quente inferior a $22{ }^{\circ} \mathrm{C}$. A precipitação pluviométrica média anual do município é de 1653,7 mm (WREGE et al., 2012).

As árvores coletadas foram processadas extraindo-se corpos de prova localizados na base e no topo da altura comercial ao longo do fuste. A determinação das propriedades físicas e mecânicas foi realizada de acordo com os ensaios de Massa Específica COPANT (461/1972), Contração COPANT (462/1972) e Flexão Estática COPANT (555/1973). Os resultados obtidos foram analisados por meio de estatística descritiva básica.

\section{RESULTADOS E DISCUSSÃO}

Conforme a classificação de densidade básica de madeiras do Forest Products Laboratory (1973), a madeira de $P$. palustris aos 39 anos de idade, cultivado na região sul do Brasil, pode ser classificada como leve (Tabela 1). Contudo, essa mesma instituição classifica a madeira dessa espécie como pesada, com densidade básica de $0,54 \mathrm{~g} . \mathrm{cm}^{-3}$, para árvores provenientes de sua região de ocorrência natural, cuja diferença está associada, provavelmente, às condições de crescimento de clima e solo.

No Brasil, são escassas as informações sobre as propriedades físicas e mecânicas dessa espécie, por isso não se tem valores de referência para comparação. A densidade básica é uma das propriedades físicas mais importantes, pois ela influencia a maioria das demais propriedades da madeira, as quais podem ser conjuntamente afetadas pelo ambiente, visto que árvores cultivadas em ambientes exóticos podem apresentar propriedades da madeira significativamente diferentes daquelas cultivadas em ambientes nativos (LATORRACA, ALBUQUERQUE, 2000).

Tabela 1. Valores médios das densidades básica, aparente e verde e dos coeficientes de retratibilidade da madeira de Pinus palustris.

\begin{tabular}{lcccc}
\hline & Propriedades & Média geral & DP & CV (\%) \\
\hline \multirow{3}{*}{ Densidade $\left({\left.\mathrm{g} . \mathrm{cm}^{-3}\right)}\right.$} & Básica & 0,346 & 0,051 & 14,75 \\
& Aparente a 0\% & 0,381 & 0,060 & 15,67 \\
& Aparente a 12\% & 0,417 & 0,064 & 15,32 \\
& Verde & 0,925 & 0,101 & 10,87 \\
\hline \multirow{2}{*}{ Contração (\%) } & Tangencial & 5,575 & 0,788 & 14,13 \\
& Radial & 3,058 & 0,611 & 19,99 \\
\hline & Volumétrica & 9,050 & 1,130 & 12,48 \\
\hline
\end{tabular}

DP: desvio padrão; CV: coeficiente de variação.

Com base no valor médio encontrado para o coeficiente de anisotropia (Tabela 1) a madeira de $P$. palustris pode ser considerada de qualidade normal (1,5-2,0), segundo o critério de classificação de madeiras quanto ao fator anisotrópico (DURLO, MARCHIORI, 1992). Assim, pode-se destacar que sua estabilidade dimensional é semelhante às madeiras de outras espécies de pinus, de araucária, peroba-rosa, teca, entre outras, conforme os autores.
A resistência mecânica da madeira de $P$. palustris foi semelhante a encontrada por outros autores para outras espécies de pinus plantadas no sul do país com menor idade (Tabela 2). Como de 58,30 MPa (MOR) e $8589,71 \mathrm{MPa}$ (MOE) para P. elliottii com 21 anos de idade (LUCAS FILHO, 2012), 45,3 e 56,3 MPa (MOR) e 6.318 e $6.628 \mathrm{MPa}(\mathrm{MOE})$ para P. patula e $P$. taeda com 12 anos de idade (MUSTEFAGA et al., 2019).

Tabela 2. Valores médios do módulo de ruptura (MOR) e do módulo de elasticidade (MOE) para o ensaio de flexão estática da madeira de Pinus palustris a $12 \%$ de umidade.

\begin{tabular}{|c|c|c|}
\hline \multicolumn{3}{|c|}{ Flexão estática } \\
\hline & MOR & MOE \\
\hline Média geral (MPa) & 60,81 & 6698,22 \\
\hline Desvio padrão (MPa) & 24,46 & 3163,88 \\
\hline Coeficiente de variação (\%) & 40,22 & 47,23 \\
\hline
\end{tabular}


De modo geral, o uso de madeiras de densidade leve a média é recomendado para a produção de móveis maciços ou sarrafeados, painéis MDF (medium density fiberboard) ou MDP (medium density particleboard), esquadrias (janelas, portas, etc.), lambris, etc. (MORAES NETO et al., 2009). Assim, diante dos resultados obtidos para as propriedades físicas e mecânicas nesta pesquisa, $P$. palustris pode ser considerada uma espécie potencial para diversificação dos plantios florestais comerciais da região sul brasileira.

\section{CONCLUSÕES}

A densidade básica média da madeira de $P$. palustris foi de $0,35 \mathrm{~g} . \mathrm{cm}^{-3}$, classificada como leve.

Os valores encontrados de retratibilidade e o coeficiente de anisotropia apontaram para uma madeira dimensionalmente estável.

A resistência mecânica da madeira dessa espécie mostrou-se compatível com outras espécies de pinus cultivadas na região sul brasileira.

\section{REFERÊNCIAS}

BERENHAUSER, H. Pinus palustris, uma espécie pouco conhecida no Brasil. Floresta, Curitiba, v. 3, n. 1, p. 35-36, 1971.

BOYER, W. B. Pinus palustris Mill. longleaf pine. p. 405412. Silvics of North America. vol. 1, Conifers. USDA Forest Service Handbook 654, Washington, DC. 1990.

DORMAN, K. W. The genetics and breeding of southern pines. Washington, D.C.: US.Dept. of Agriculture, Forest Service, 1976.

DURLO, M. A., MARCHIORI, J. N. C. Tecnologia da madeira: retratibilidade. Santa Maria: CEPEF/FATEC, 33 p, 1992 (Série Técnica, 10).
FOREST PRODUCTS LABORATORY. Standard terms for describing wood. USDA. Forest Service. Forest Products Laboratory Research Paper. Madison, v.0171, p.1 - 10, 1973. Disponível em: https://www.fpl.fs.fed.us/products/publications/specific_p ub.php?posting_id=17629\&header_id=p. Acesso em: 18 out. 2020.

LATORRACA, J. V. de F., ALBUQUERQUE, C. E. C. de. Efeito do rápido crescimento sobre as propriedades da madeira. Floresta e Ambiente, Seropédica, v. 7, n. 1, p. 279-291, 2000.

LUCAS FILHO, F. C. Efeito combinado do teor de umidade e da massa específica na resistência e rigidez da madeira de Pinus elliottii. Floresta, Curitiba, v. 42, n.3, p.519-526, 2012.

MORAES NETO, S. P. de, TELES, R. F., RODRIGUES, T. O., VAlE, A. T., SOUZA, M. R. de. Propriedades mecânicas da madeira de cinco procedências de Pinus caribaea var. hondurensis implantadas no cerrado do Distrito Federal, DF. Planaltina, DF: Embrapa Cerrados, 2009.

MUStefaGA, E. C., HILliG, E., TAVARES, E. L., SOZIM, P. C. L., RUSCH, F. Caracterização físicomecânica da madeira juvenil de Pinus. Scientia Forestalis, Piracicaba, v.47, n.123, p.472-481, 2019.

SHIMIZU, J. Y. Testes preliminares de procedências de Pinus palustris Mill. no Sul do Brasil. In: CONGRESSO FLORESTAL BRASILEIRO - SILVICULTURA, $3^{\circ}$, 1978, Manaus. Anais [...]. São Paulo: Silvicultura, 1979, v.2, n.14, p.130-132.

WREGE, M. S., STEINMETZ, S., REISSER JÚNIOR, C., ALMEIDA, I. R. de. Atlas Climático da Região Sul do Brasil: Estados do Paraná, Santa Catarina e Rio Grande do Sul. 2. ed. Brasília: Embrapa, 2012. 